\title{
RADIATION SYNTHESIS OF POLY VINYLPYRROLIDONE AND POLYACRYLAMIDE HYDROGELS AS SOIL CONDITIONER.
}

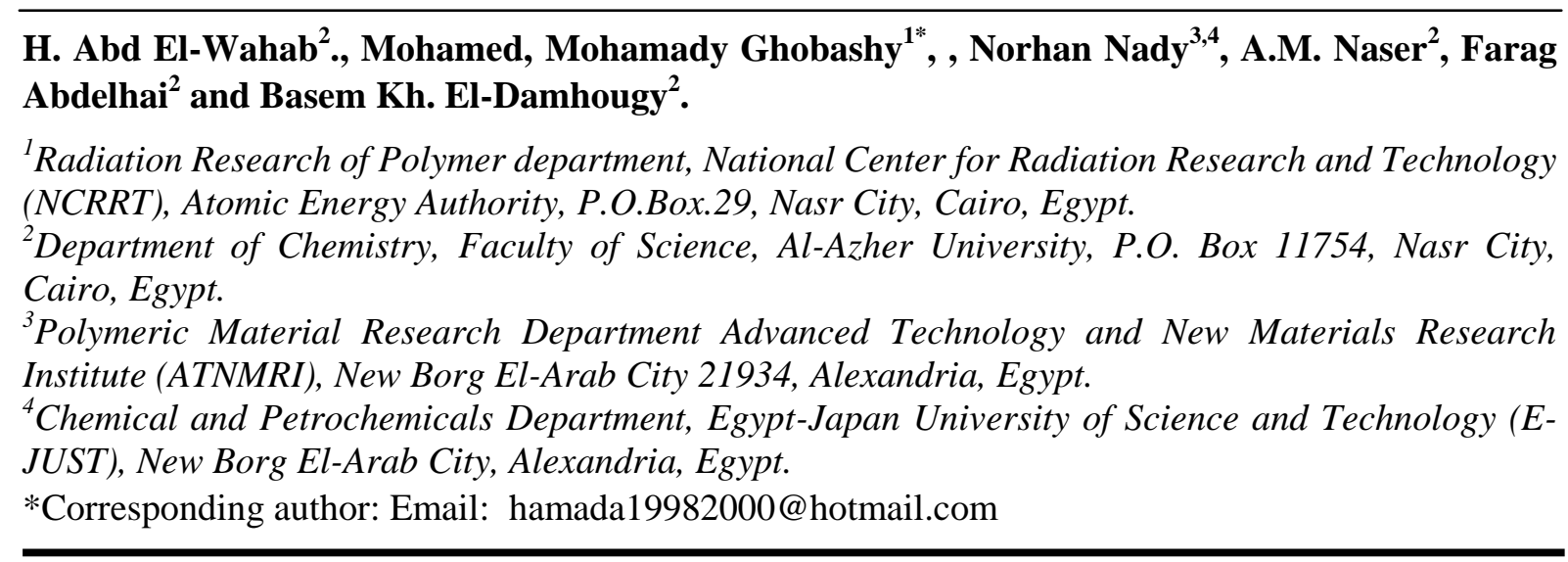

\begin{abstract}
The important to synthesize a lower toxicity hydrogel with high biocompatibility to use in agricultural field. In this regard, the polymerization reaction of Poly vinylpyrrolidone and Poly acrylamide (PVP/PAAm) hydrogel has been obtained after exposed the copolymer and monomer mixture to dose of $30 \mathrm{kGy}$. The equilibrium swollen of the obtained hydrogel was $25(\mathrm{~g} / \mathrm{g})$ after $60 \mathrm{~min}$., has an advantage to use as soil conditioner. The hydrogels samples have been prepared in weight ratios 25:75, 50:50 and 75:25 (wt:wt). The crosslink density combining this with measurements of the swelling degree was depend on the copolymer concentration of two polymers. The morphology and the chemical structure of the PVP/PAAm hydrogels was characterized using Scanning electron microscope (SEM), Fourier transform infrared spectroscopy technique (FTIR), and X-ray diffraction (XRD). The PVP/PAAm hydrogels with ratio (75/25) were selected to evaluate the effectiveness on water holding capacity (WHC) in heavy metals and alkaline soil $\mathrm{pH}>9$ for plating sunflower (Helianthus annuиs).
\end{abstract}

Key word: Radiation synthesis; polymerization; hydrogel; sunflower; soil conditioner

\section{INTRODUCTION}

The definition of hydrogels is made according to the main properties and raw materials. Hydrogels are materials with viscoelastic properties and network structure caused by cross-linker and the solvent, respectively. Recently, hydrogel materials have been pervaded in daily life in different forms depending on their needed application such as soap, shampoo, toothpaste, hair gel and contact lenses [1]. There are some advance industrial applications of hydrogels such as oil recovery, pharmaceutical, agriculture, textile, and water treatment. Therefore, gel materials have become one of the most popular materials in our daily life. Hydrogels are different according to needed application such as the gels for body care is not same as that for agriculture or oil recovery application. A new class of hydrogels was discovered and reported first by the U.S. Department of Agriculture, which they called superabsorbent polymer/hydrogel (SAP/SAH) materials. They are reporting it as loosely cross-linked hydrophilic polymers that can swell, absorb and retain a large volume of water or other biological fluids [2-5], and also the absorbed fluids hardly removed under some pressure [6]. In other words this class of materials can be defined as an advance form of hydrogels or can be said to be hydrogels with high potential to absorb water or other fluids and swell 1000 times than of their dried weight. This kind of material can be applied in areas where high absorption is needed to enhance the absorption capacity, biodegradability, reduce price of hydrogels and/or SAP and make it more suitable for some application. Hydrogels and SAP have been filled with natural and synthesized filers in the last decade; the product 
is then called a hydrogel or a SAP composite. Many scientists defined hydrogel based on different aspects.

Hydrogels are a three-dimensional network, which can be formed by crosslinking polymer chains. The cross-linking of these polymers is a result of covalent, hydrogen bonding, van der Waals forces or physical entanglements [7]. Another scientist said that hydrogels are threedimensional matrices, viscoelastic solid-like materials which are comprised of an elastic cross-linked network and solvent as its main components. The solid-like gels are a result of entrapment and adhesion of the liquid in the large solid 3D surface area matrix. The formation of solid matrix is a result of crosslinking polymer strands (macro) of molecules due to physical or chemical forces [1,8-12]. Hydrogels can be designed with controllable responses as to shrink or expand with changes in external environmental conditions [13]. It may respond uniquely to changes in external environmental conditions such as ionic strength [14], electromagnetic radiation [15], $\mathrm{pH}$ [1620], and temperature [16, 18, 21-23]. The extent of swelling or de-swelling in response to the changes in the external environment of the hydrogel could be so drastic that the phenomenon is referred to as volume collapse or phase transition [24-26] and such hydrogels are called stimuli sensitive hydrogels.

The aim of this study is to investigate the swelling properties of poly vinyl pyrilldone and polyacrylamide hydrogels. Hydrogels have been prepared by gamma irradiation techniques at room temperature $\left(25^{\circ} \mathrm{C}\right)$. The influence of copolymer composition on gel fraction and swelling behavior was investigated.

\section{EXPERIMENTAL}

\section{Materials and methods}

\subsection{Materials}

Polyvinylpyrrolidone (PVP) has average M.W 100,000 (Acros, Belgium). Acrylamide (Am) monomer 99\% supplied from SigamAldrich Co.

\subsection{Radiation preparation of PVP/PAAm hydrogels}

The hydrogels of PVP and Am have been formed using radical polymerization reaction initiated by gamma radiation. A solution of PVP was stirred and heated to $80{ }^{\circ} \mathrm{C}$ for $10 \mathrm{~min}$. Viscous solution is formed and then adds to Am with three weight ratio $(25-75,50-50$ and 75-25) wt-wt. kept at $10 \%$ copolymer concentration. The radiolysis of water creation radical that transfer to monomers to form macroradicals molecules. Mechanism of radical polymerization is driven by recombination of two macroradical molecules forming dimer, trimmer and finally crosslinked hydrogel will obtained. the (PVP/PAAm) solutions were placed into polyethylene bags. The bags were closed and irradiated at irradiation doses 30 kGy from 60-Co $\gamma$-source at National Center for Radiation Research and Technology, Atomic Energy Authority, Cairo, Egypt, at dosage rate; $1.66 \mathrm{kGy} / \mathrm{h}$. The obtained hydrogel was cut into granules and dried in air after washing and remove unreacted part at room temperature and stored in dry conditions for further studies.

\subsection{Swelling degree determined}

A certain weight of dried hydrogel ( $\mathrm{W}_{\mathrm{o}}$ ) was put in distilled water at ambient temperature. At a period of time intervals, the weight of swollen hydrogel was recoded $\left(\mathrm{W}_{\mathrm{t}}\right)$. The degree of swelling $(\mathrm{g} / \mathrm{g})$ was calculated from the following equation.

$$
\mathbf{S}(\mathbf{g} / \mathbf{g})=\left(\mathbf{W}_{\mathrm{o}}-\mathbf{W}_{\mathrm{t}}\right) / \mathbf{W}_{\mathrm{o}}
$$

The measurement of swelling was continued until maximum weight was reached, it called swelling equilibrium.

\subsection{Pilot study on the application of the prepared hydrogel as soil conditioner in agriculture proposed}

Soil containing heavy metals and ability of hydrogel to overcomes the heavy metals stress on the germination of plant has been evaluated. Effect of heavy metals stress was performed by encourages sunflower (Helianthus annuus). 
Plants will grow in stress of heavy metals conditions. Two phases are compared, one is soil without hydrogel(control) and other treatments with (PVP/PAAm) hydrogel. In Phase 1 the test soil is untreated with hydrogel. In Phase 2, added $10 \mathrm{gm}$ of dry PVP/PAAm mixed with $2000 \mathrm{gm}$ of soil. In addition, the dispersed dry hydrogel powder on moisture soil is benefit of absorb water gain from the rainfalls. The swelling of PVP/PAAm hydrogel in moisture soil leads to increase of soil porosity and leads to good ventilation reflected on the growth of the plant.

\subsection{Instrumentation}

The Fourier Transform Infrared (FTIR) spectra were performed by using Vertex 70 . FTIR spectrometer was supplied from, BrukerOptik GmbH, Ettlingen, Germany, the measurement ranged was from $4000-400 \mathrm{~cm}^{-1}$, with a resolution of $4 \mathrm{~cm}^{-1}$. Scanning electron microscope (SEM, JEOL, and model JSM5310) imaging was done at an accelerating voltage of $30 \mathrm{kV}$. In this specific case, the hydrogel samples were first immersed in distilled water over night until reaching equilibrium swelling, and then then the sample was freeze dried and was analyzed by SEM. Attenuated Total Reflection (ATR) mode was used. X-ray diffraction (XRD) was carried out using a Phillips X'Pert Pro MPD diffractometer with copper as source of $\mathrm{x}$-ray at $\lambda=1.5 \mathrm{~nm}$. The voltage, the current, and the wavelength of the X-ray source were $30 \mathrm{~mA}, 20 \mathrm{kV}$.

\section{RESULTS AND DISCUSSION}

\subsection{Prefer agriculture applications (soil conditioner) based on swelling property in water and moisture soil.}

The ability of hydrogels to absorb water could be attributed to the existence of hydrophilic groups such as carboxyl, hydroxyl and amide groups in their matrices. Polyvinylpyrrolidone and polyacrylamide contain hydrophilic groups in their backbone structure, viz. amide, hydroxyl and carbonyl groups, respectively. Therefore, these materials are considered as better materials for manufacturing hydrogels for using as soil conditioner. The copolymerized by means of forming interpenetrating networks of poly(acrylamide) and poly (vinyl pyrrolidone). Figure 1, shows that the swelling degree of the hydrogels samples reach maximum after 60 min. The swelling degree of the three copolymeric hydrogel is depending on the ratio of PVP contents. It is cleared that in the presence of hydrophilic hydrogel matrices increases the water absorption capacity. On the other hand, the intramolecular H-bonds between and intermolecular H-bonds with the two hydrogels of (PVP and PAAm). Thus the degree of swelling decreased with increased (Am) content was attributed to reduce the number of hydrophilic hydrogel and increasing H-bonds.

Table 1 shows the swelling degree of (PVPPAAm) hydrogel is increased with the content of PVP increased. This is due to that the Am Segments provide $\mathrm{H}$ bonds that lead to decreasing the swelling degree. The greater swelling degree and gel fraction were founded high in the weight ratios of (75-25).

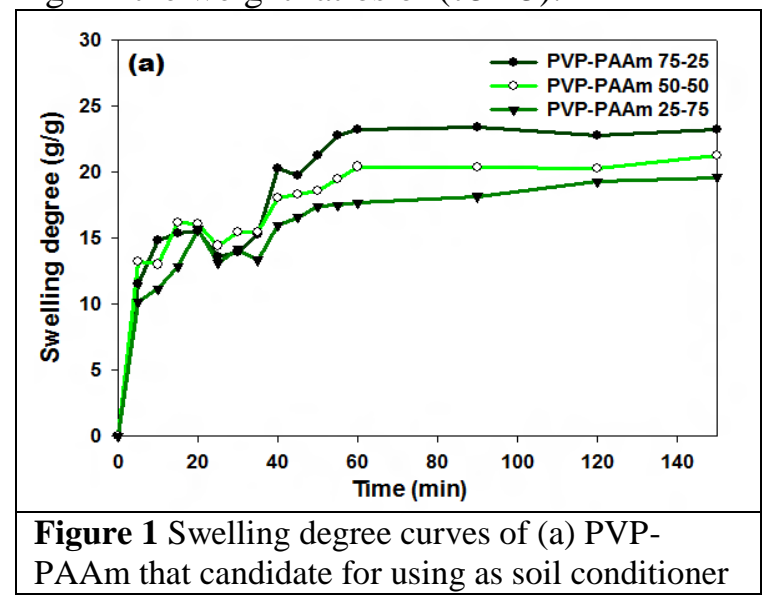

Table 1 present the swelling and gel fraction of three IPN hydrogel samples

\begin{tabular}{|c|c|c|c|c|}
\hline $\begin{array}{c}\text { Code } \\
\mathrm{s}\end{array}$ & PVP(gm) & Am $(\mathrm{gm})$ & $\begin{array}{c}\text { Swelling } \\
(\mathrm{g} / \mathrm{g})\end{array}$ & $\begin{array}{c}\text { Gel fraction } \\
(\%)\end{array}$ \\
\hline 1 & 1.5 & 0.5 & $\mathrm{rr}, \mathrm{\vee}$ & 91.9 \\
\hline 2 & 1 & 1 & 17.7 & 72.3 \\
\hline 3 & 0.5 & 1.5 & 16.6 & 68.9 \\
\hline
\end{tabular}

Figure 2 show the swelling of (PVPPAAm) in weight ratios of (75-25) in moisture soil ranged from $2.5,5,10,15$ and $20 \%$. It is 
cleared that the swelling degree is moisture depended and promise how the dry hydrogel capable to absorb a high amount water from moisture soil after $60 \mathrm{~min}$. The capacity of hydrogels to absorb water from moisture soil would increase the efficient of the rainfalls exploitation and water from sprinkling irrigation.

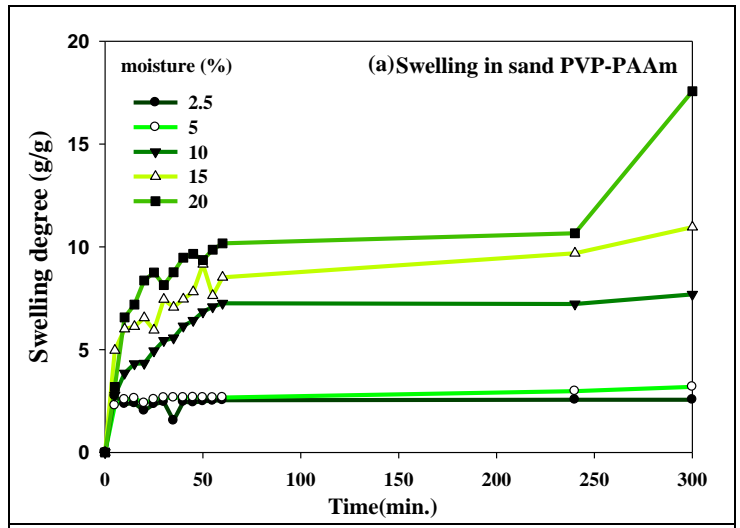

Figure 2 Swelling degree in the moisture soil of the PVP/PAAm hydrogel sample for uses as soil conditioner.

Figure 3 show the water retention of swelled hydrogel of the (PVP-PAAm) in weight ratios of (75-25). The hydrogel lost most of the water content after two days. This suggested that PVP/PAAm is favorable for the soil conditioner applications. This could be attributed to the hydrophilic chains of PVP, which created a hydrophilic segment, thus this could be minimizing the loss of water from the hydrogels.

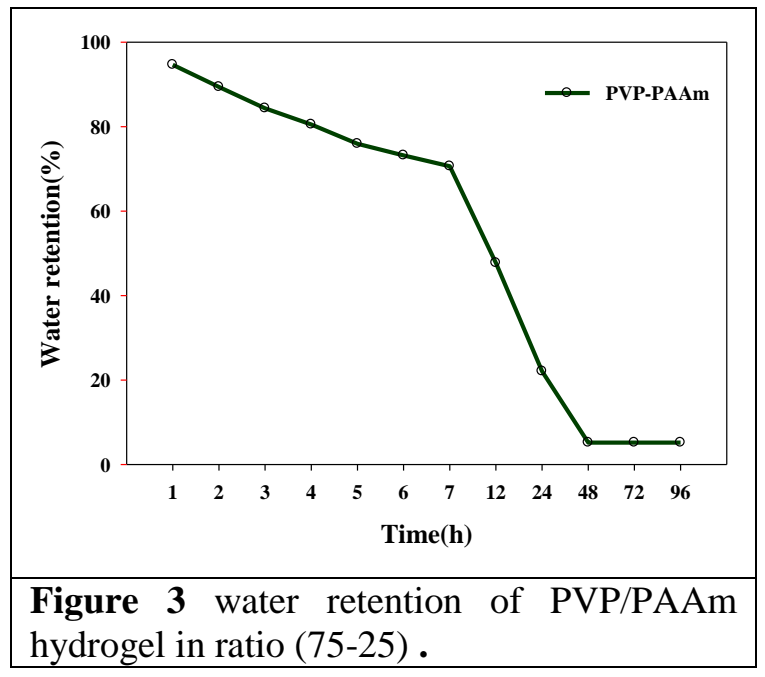

\subsection{Chemical structure performed by FTIR of the three IPN hydrogel}

FTIR spectrum of PVP/PAAm, is show in Figure 4. FTIR spectrum shows broad peak at $1638 \mathrm{~cm}^{-1}$, attributing to the carbonyl group of the acrylamide and pyrrolidone segments. An extremely broad peak due to hydrogen bonds between hydroxyl groups $(\mathrm{O}-\mathrm{H})$ and functional groups of PAAm and PVP appears at $3448 \mathrm{~cm}^{-}$ ${ }^{1}$. Consequently, it is concluded that the PAAm and PVP polymers were successfully polymerized as copolymer hydrogel.

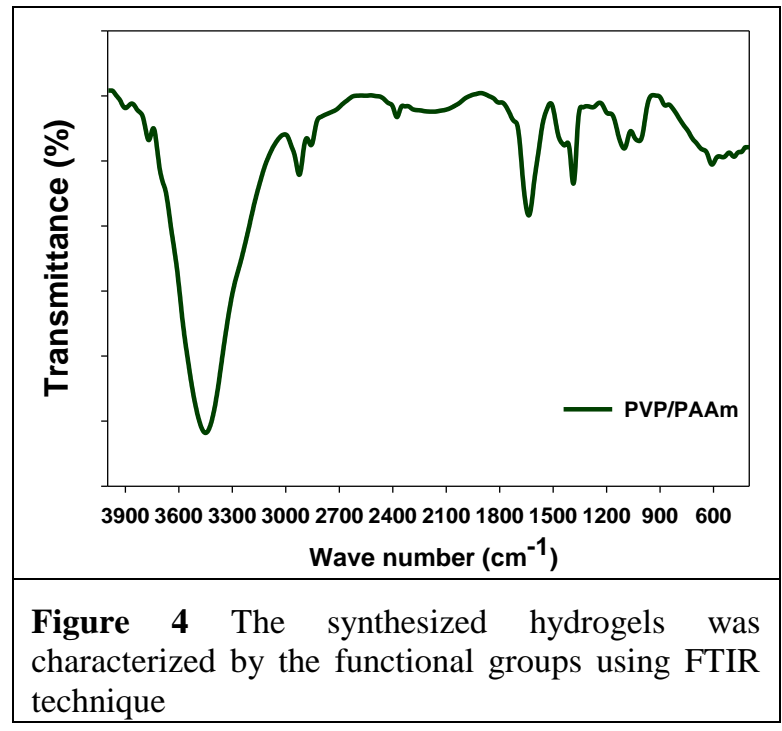

\subsection{Scanning electron microscopy}

Figure 5 show scanning electron microscopic of the (PVP-PAAm) in weight ratios of (75-25). Clearly shows the presence of differ quantities of interconnected pores indicating a difference in crooslinked density. A large quantity of interconnected pores, indicating the high gel fraction was obtained. The PVP-PAAm hydrogel had high porosity structure lead to faster swelling as proved in Figure 1. The interconnected pores allowed the hydrogel to absorb water rabidly. Furthermore, entanglement of PVP and PAAm matrices led to increase the net points and pores units, which favored to absorb water. 


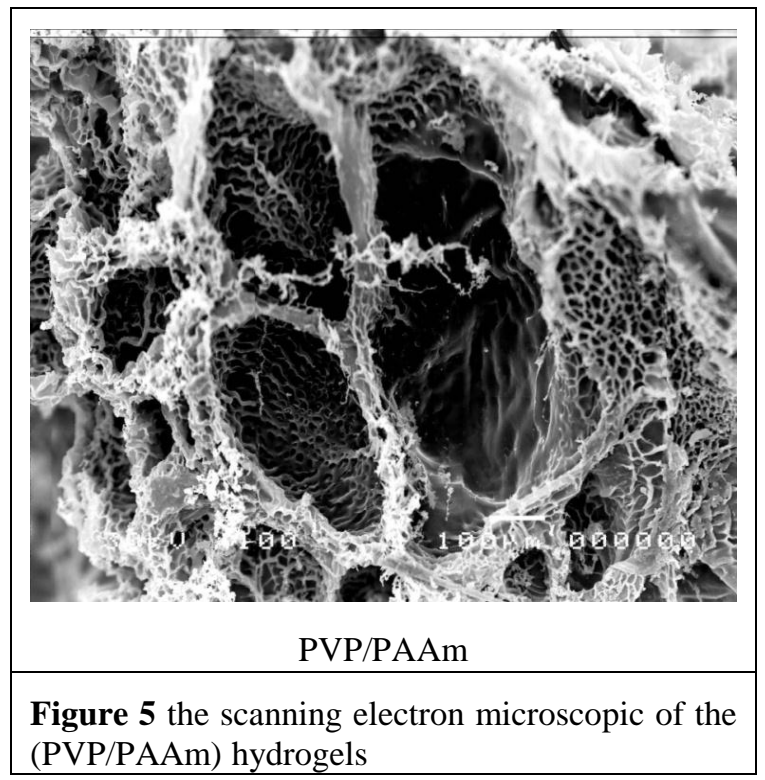

\subsection{XRD Analysis of IPN hydrogels}

PVP and PAAm are semicrystallineas observed in Figure 6 . The three sharp diffraction peaks at $14.3^{\circ}, 15.7^{\circ}$ and $22.1^{\circ}$, the semicrystallinity is could due to inter and intramolecular H-bonds. XRD pattern of (PVP/PAAm) hydrogel given one brooding peak at $26.5^{\circ}$.

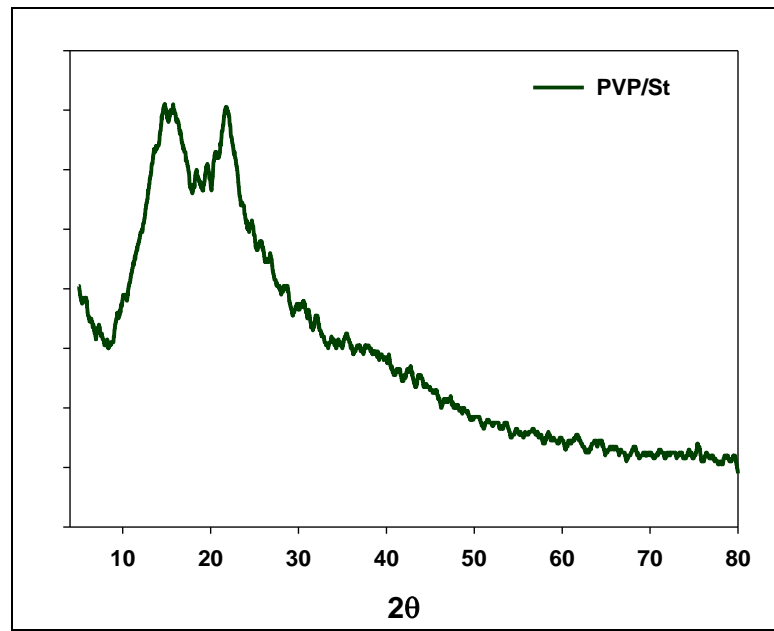

Figure 6 XRDpatterns of PVP/PAAm hydrogel samples

\subsection{Effect of different soil conditioner in heavy ions stress condition on sunflower plant}

Durations of sunflower growth stages for each treatment are shown in Figure 7. Our study showed that the PVP/PAAm hydrogel influence the duration of sunflowers seedling stage in soil salinity. However, for the mature stages, the durations for PVP/PAAm treatments increased by $39.89 \%$ on average compared with the control treatments. Figure 7 showed that heavy metals stress significantly prolonged the duration of the sunflower. Consequently, the average duration of bud stages and total growth period for the treatments increased in order PVP/PAAm>control.
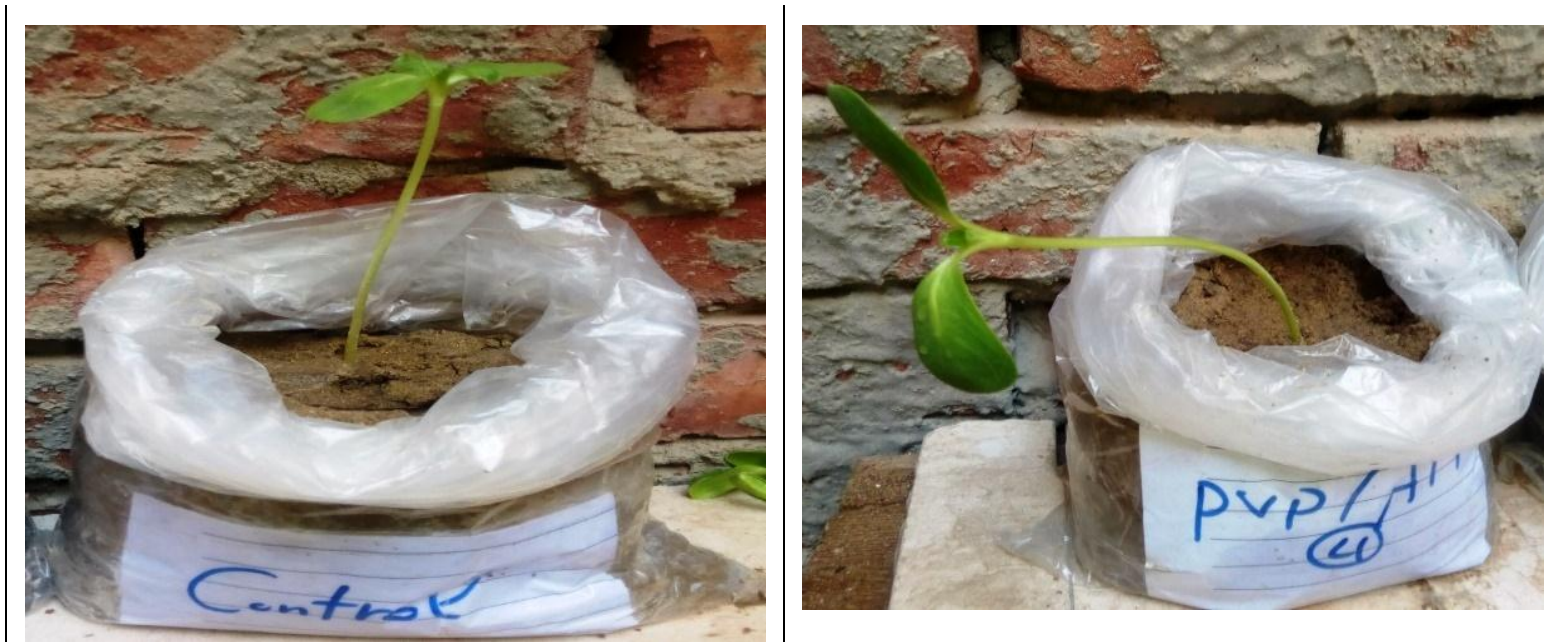

Figure 7 the image of planting in control soil and treatment soil with PVP/PAAm hydrogel sample. 


\section{CONCLUSION}

Several hydrogels were synthesized by crosslink copolymerization of acrylamide and poly vinyl pyrrolidone. The semi crystallinity of hydrogel sample in the copolymer gel matrix was observed by XRD. Swelling degree of the hydrogels were evaluated. The degree of swelling increased with increased (PVP) content due to increase the number of hydrophilicity. Semi-crystalline (PVP/PAAm) not only tangled with poly acrylamide and poly vinyl pyrrolidone chains though numerous hydrogen bonds, but formed intramolecular $\mathrm{H}$ bonds as well. The obtained hydrogels are capable to absorb water from soil moisture that engage for using as soil conditioner. Hydrogel synthesized with 75 PVP and 25 PAAm shows the highest swelling. This hydrogel also showed high significant influence in the growth of sunflowers in soil alkalinity adversely affects the quality and productivity of plants. Sunflower growth is planting in soil has high amounts of heavy metals and $\mathrm{pH}$ was 8.9. Our study proved that the PVP/PAAm hydrogel a significant influence on sunflower phenology rather than un treatment.

\section{REFERENCES}

1. Mailra, N. (2005). Supramolecular gels: Function and uses. Chem. Soc. Rev, 34, 821-836.

2. Zhang, J., \& Wang, A. (2007). Study on superabsorbent composites. IX: synthesis, characterization and swelling behaviors of polyacrylamide/clay composites based on various clays. Reactive and Functional Polymers, 67(8), 737-745..

3. Wu, L., Liu, M., \& Liang, R. (2008). Preparation and properties of a double-coated slow-release NPK compound fertilizer with superabsorbent and water-retention. Bioresource technology, 99(3), 547-554..

4. Guilherme, M. R., Reis, A. V., Takahashi, S. H., Rubira, A. F., Feitosa, J. P., \& Muniz, E. C. (2005). Synthesis of a novel superabsorbent hydrogel by copolymerization of acrylamide and cashew gum modified with glycidyl methacrylate. Carbohydrate Polymers, 61(4), 464-471.
5. Liang, R., Yuan, H., Xi, G., \& Zhou, Q. (2009). Synthesis of wheat straw-g-poly (acrylic acid) superabsorbent composites and release of urea from it. Carbohydrate Polymers, 77(2), 181$187 .$.

6. Tang, Q., Wu, J., Sun, H., Fan, S., Hu, D., \& Lin, J. (2008). Superabsorbent conducting hydrogel from poly (acrylamide-aniline) with thermosensitivity and release properties. Carbohydrate polymers, 73(3), 473-481.

7. Qiu, Y., \& Park, K. (2001). Environmentsensitive hydrogels for drug delivery. Advanced drug delivery reviews, 53(3), 321-339..

8. Karadağ, E., Üzüm, Ö. B., Saraydin, D., \& Güven, O. (2005). Dynamic swelling behavior of $\gamma$-radiation induced polyelectrolyte poly (AAm-co-CA) hydrogels in urea solutions. International journal of pharmaceutics, 301(1-2), 102-111.

9. Patachia, S., Valente, A. J., \& Baciu, C. (2007). Effect of non-associated electrolyte solutions on the behaviour of poly (vinyl alcohol)-based hydrogels. European Polymer Journal, 43(2), 460-467..

10. Singh, T. R. R., McCarron, P. A., Woolfson, A. D., \& Donnelly, R. F. (2009). Investigation of swelling and network parameters of poly (ethylene glycol)-crosslinked poly (methyl vinyl ether-co-maleic acid) hydrogels. European Polymer Journal, 45(4), 1239-1249..

11. Xuequan, L., Maolin, Z., Jiuqiang, L., \& Hongfei, H. (2000). Radiation preparation and thermo-response swelling of interpenetrating polymer network hydrogel composed of PNIPAAm and PMMA. Radiation Physics and Chemistry, 57(3-6), 477-480..

12. Liu, P., Peng, J., Li, J., \& Wu, J. (2005). Radiation crosslinking of CMC-Na at low dose and its application as substitute for hydrogel. Radiation Physics and Chemistry, 72(5), 635-638.

13. Park, K., Shalaby, W.S., Park, H. (1993). Biodegradable Hydrogels for Drug Delivery,Technomic Publishing Company, Inc., Basel, pp. 1-12, 35-66.

14. Siegel, R. A., \& Firestone, B. A. (1988). pHdependent equilibrium swelling properties of hydrophobic polyelectrolyte copolymer gels. Macromolecules, 21(11), 3254-3259..

15. Grodzinsky, A. J., \& Weiss, A. M. (1985). Electric field control of membrane transport and 
separations. Separation and Purification Methods, 14(1), 1-40.

16. Hendri, J., Hiroki, A., Maekawa, Y., Yoshida, M., \& Katakai, R. (2001). Permeability control of metal ions using temperature-and $\mathrm{pH}$ sensitive gel membranes. Radiation Physics and Chemistry, 60(6), 617-624..

17. Qiu, Y., \& Park, K. (2001). Environmentsensitive hydrogels for drug delivery. Advanced drug delivery reviews, 53(3), 321-339..

18. Ng, L-T., Arsenin, A., Nguyen D., Proc. Radiat. Tech., 3, 669, 2003.

19. Brannon-Peppas, L., \& Peppas, N. A. (1989). Solute and penetrant diffusion in swellable polymers. IX. The mechanisms of drug release from $\mathrm{pH}$-sensitive swelling-controlled systems. Journal of Controlled Release, 8(3), 267-274..

20. Kou, J. H., Amidon, G. L., \& Lee, P. I. (1988). pH-Dependent Swelling and Solute Diffusion Characteristics of Poly (Hydroxyethyl Methacrylate-CO-Methacrylie Acid) Hydrogels. Pharmaceutical research, 5(9), 592597..

21. Bae, Y. H., Okano, T., \& Kim, S. W. (1989). Insulin permeation through thermo-sensitive hydrogels. Journal of Controlled Release, 9(3), 271-279..

22. Dong, L.C., Hoffman, A.S., ACS Symposium Series 350, Russo, P., ed., American

Chemical Society, Washington, DC, pp. 236, 1987.

23. Freitas, R. F., \& Cussler, E. L. (1987). Temperature sensitive gels as extraction solvents. Chemical Engineering Science, 42(1), 97-103..

24. Tanaka, T. (1981). Gels. Scientific American, 244(1), 124-S..

25. Tanaka, T., ACS Symposium Series 480, Harland, R.S., Prudhomme, R.K., eds.,

American Chemical Society, Washington, DC, pp. 1-22, 1992.

26. Grosberg, A. Y., \& Nechaev, S. K. (1991). Topological constraints in polymer network strong collapse. Macromolecules, 24(10), 27892793..

27 Chen, Y., Liu, S., \& Wang, G. (2007). Synthesis and physicochemical properties of graft copolymer of corn starch and acrylamide. Polymer composites, 28(1), 47-56. 